Educación Física y Ciencia, vol. 19, n 1, e020, junio 2017. ISSN 2314-2561

Universidad Nacional de La Plata.

Facultad de Humanidades y Ciencias de la Educación.

Departamento de Educación Física

\title{
Aptitud cardiorrespiratoria y adiposidad frente al nivel de actividad física
}

\author{
Cardiorespiratory fitness and adiposity against physical activity level
}

\section{Carlos Alberto Ramos Parrací *; Constanza Palomino Devia *; Nelson Rodríguez Arias *}

*Universidad del Tolima, Colombia | caramosp@ut.edu.co; cpalominod@ut.edu.co; nrodriguezarias@gmail.com

\section{PALABRAS CLAVE}

Actividad física

Estilo de vida

Aptitud física

Adiposidad

\section{KEYWORDS}

Physical Activity

Life style

Physical fitness

Adiposity

\section{RESUMEN}

Con el objetivo de determinar el comportamiento de la Aptitud Cardiorrespiratoria y la adiposidad frente al nivel de actividad física de la población adulta de la ciudad de Neiva, se evaluaron 972 sujetos entre 18 a 75 años de edad. Estudio Descriptivo Correlacional. Los datos se analizaron en SPSS-23 e InfoStat/Profesional 1,2. La metodología partió de la descripción de variables, posteriormente el grado de asociación entre ellas (coeficiente de correlación de Pearson), los grupos conformados se compararon (Prueba ANOVA y comparación multiple LSD Fisher); por último, se estableció el grado de agrupamiento entre las variables (Prueba Average Linkage). Los resultados evidenciaron diferencias significativas en índice de masa corporal (IMC), Frecuencia Cardiaca Reposo (FCR) y Consumo Máximo de Oxigeno ( $\mathrm{VO}_{2}$ máx.), entre activos e inactivos; asociación del 5\%, entre el IMC con FCR y VO $\mathrm{V}_{2}$ máx, índice cinturacadera (ICC) y porcentaje grasa corporal (\%GC); del ICC con \%GC, $\mathrm{VO}_{2}$ máx, Tensión Arterial Sistólica (TAS) y Diastólica (TAD); del \%GC con FCR, TAD y $\mathrm{VO}_{2}$ máx; la FCR con $\mathrm{VO}_{2}$ máx; la TAS con TAD y $\mathrm{VO}_{2}$ máx rechazando la hipótesis de independencia. Concluyendo que los indicadores de adiposidad y aptitud cardiorrespiratoria evidencian la combinación de factores de riesgo de enfermedades de índole hipocinético en la población.

\section{ABSTRACT}

The aim of this study is to determine the behaviour of cardiorespiratory aptitude and adiposity against the physical activity of the adult population in Neiva Colombia. 972 subjects between 18 to 75 years of age were evaluated. This is a descriptive correlational study. Data were analyzed in SPSS-23 and InfoStat / Professional 1,2. The methodology departed from the description of variables, then the degree of association between them (Pearson correlation coefficient), the shaped groups (ANOVA test and LSD multiple comparison Fisher) were compared; and finally, the degree of clustering among the variables (Test Average linkage) was established. The results evidenced meaningful differences in the body mass index (BMI), resting heart rate (RHR) and maximum oxygen consumption $\left(\mathrm{VO}_{2}\right.$ máx.), between active and inactive; $5 \%$ association among the BMI with RHR and $\mathrm{VO}_{2}$ máx. Waist Hip Ratio (WHR) and body fat percentage (BF\%); of WHR with $\mathrm{BF} \%, \mathrm{VO}_{2}$ máx, systolic blood pressure (SBP) and diastolic (DBP); of the $\mathrm{BF} \%$ with RHR, DBP and $\mathrm{VO}_{2}$ máx; the RHR with $\mathrm{VO}_{2}$ máx; the SBP with DBP and $\mathrm{VO}_{2}$ máx rejecting the independence hypothesis. As a conclusion, adiposity indicators and cardiorespiratory aptitude are a combination of disease risk factors of hypokinetic in the population. 


\section{Introducción}

La práctica de la actividad física constituye uno de los principales triunfos de un estilo de vida saludable y de una verdadera protección y promoción de la salud. En las últimas dos décadas, numerosos estudios científicos realizados hasta nuestros días han demostrado que la actividad física regular proporciona importantes beneficios en la salud (Blacklock, Rhodes \& Brown, 2007; Buchheit et al., 2006; Haskell et al., 2007), quedando demostrado como la realización de actividad física mejora la salud de la persona y coadyuva al tratamiento de enfermedades como la obesidad, la aterosclerosis y las enfermedades cardiovasculares, la hipertensión, algunas enfermedades respiratorias, la diabetes no insulino dependiente, el cáncer, las enfermedades reumatoides y la depresión, entre otras (Haskell et al., 2007), siempre y cuando obedezca a unos criterios de tipo, duración, intensidad, frecuencia y cadencia, y se adapte a las posibilidades de cada individuo. Como consecuencia de ello, la actividad física ha sido promocionada últimamente desde las autoridades públicas y otras organizaciones como un instrumento efectivo para prevenir hábitos nocivos para la salud, siendo señalada la condición física y el ejercicio como una de las diez áreas de mayor importancia en la salud pública y uno de los desafíos futuros importantes (Powell \& Paffenbarger 1985), debido a los beneficios evidentes de su práctica para la salud física, social y mental, como ha sido también manifestado y reconocido por la población en general (Malmberg, Miilunpalo, Pasanen, Vuori \& Oja, 2005; Pahor et al., 2006).

Sin embargo, es importante resaltar, que la cantidad y calidad de ejercicio que se necesita para alcanzar los beneficios relacionados con la salud pueden ser diferentes de lo que se recomienda para los beneficios de fitness. Está claro, que reducir los niveles de actividad física (en particular la intensidad) de lo recomendado, puede disminuir el riesgo de ciertas enfermedades degenerativas crónicas y mejorar la salud metabólica, sin embargo, no puede ser la suficiente cantidad o calidad para mejorar el $\mathrm{VO}_{2}$ máx (Osei-Tutu \& Campagna, 2005). Un claro ejemplo de ello, es la aptitud metabólica, término introducido para describir el estado de los sistemas metabólicos y las variables predictoras del riesgo de diabetes y enfermedades cardiovasculares, la cual puede ser favorablemente alterada por una mayor actividad física o ejercicio de resistencia regular, sin la exigencia de un aumento relacionado con el incremento del $\mathrm{VO}_{2}$ máx (Després et al., 1991). Del mismo modo, y siendo coherentes con lo expuesto, el American College of Sports Medicine (ACSM) reconoce los beneficios potenciales para la salud del ejercicio regular realizado con más frecuencia y durante más tiempo pero con una intensidad inferior, es decir del 55-64\% de la Frecuencia Cardiaca Máxima de Reserva (Thompson et al., 2003). En consecuencia, se ha abordado la cuestión relativa a la cantidad apropiada de actividad física necesaria para obtener beneficios para la salud en diversas enfermedades crónicas, por ejemplo, enfermedad cardiaca coronaria (Vuillemin et al., 2005), la hipertensión (ACSM, 1993), la osteoporosis (ACSM, 1995) y la obesidad y control de peso (Wing, 1999).

Siendo así, la actividad física y el ejercicio físico para la salud, deben verse dentro de una relación dosis-respuesta, en la cual los beneficios se obtienen a través de la ejecución de diversas actividades enmarcadas por los principios de calidad y cantidad (Lee, Hsieh \& Paffenbarger, 1995). Ya que se ha reconocido que muchos de éstos beneficios significativos para la salud, se obtienen al considerar por lo menos dos parámetros, el primero, sería pasar de un estado sedentario a un nivel mínimo de actividad física, y dos, que la práctica de actividad física, se ejecute con intesidades altas y/o mayor frecuencia/duración, lo que permitirían proporcionar ventajas adicionales.

Basados en estas consideraciones, el ACSM ha hecho un llamado claro, para que todos los gobiernos promuevan la actividad física como una de las estrategias para disminuir el riesgo de múltiples enfermedades e incrementar los beneficios que pueden obtener los individuos y sociedades físicamente activas. Esta organización afirma que el sedentarismo es un serio problema de salud pública, que afecta la calidad de vida de los individuos y la sociedad, generando una pérdida importante del potencial del ser humano (cualidades físicas, mentales y sociales) e incrementando los costos económicos de salud (Pate et al., 1995).

Logicamente Colombia no es ajena a esta realidad, en los últimos años las enfermedades del sistema circulatorio fueron la primera causa de mortalidad en hombres y en mujeres entre 2005 y 2015, las enfermedades isquémicas del corazón produjeron el 48,6\% (226.654) de las muertes por enfermedades del sistema circulatorio, con una tasa 
de 75,58 muertes por cada 100.000 habitantes; las enfermedades cerebrovasculares provocaron el 23,8\% (111.255) de las muertes, con tasas ajustadas por edad de 32,7 muertes por cada 100.000 habitantes. Por su parte, las enfermedades hipertensivas causaron el 10,2\% (47.417) de las muertes, con un comportamiento incremental, pasando de 15,5 a 16,9 muertes por cada 100.000 personas durante el periodo, lo cual equivale a tasas ajustadas por edad en un 9\% más altas y a 1,4 muertes adicionales por cada 100.000 habitantes (Minsalud, 2014).

La ciudad de Neiva, capital del Departamento del Huila, al suroccidente del país, con una superficie de $1.533 \mathrm{~km}^{2}$ y con 344.026 habitantes (DANE, 2009), a 2014, Neiva tenía una cobertura de aseguramiento del 93,1\% y registraba porcentajes altos de obesidad y sobrepeso en adultos de 18 a 64 años, en los que el 61,9\% de la población presenta problemas de sobrepeso, el $74 \%$ de obesidad abdominal y el $82 \%$ sedentarismo, según el informe del Estado Nutricional en el Huila del año 2014 (Minsalud, 2014), lo que se traduce, en una situación de gran interés para analizar y que permita identificar el comportamiento de la aptitud cardiorrespiratoria y la adiposidad frente a los niveles de actividad física en la población de Neiva y los factores relacionados con éste, con el fin de fundamentar y reorientar las prácticas de prevención, promoción de la salud e intervención temprana respecto a esta problemática.

\section{Metodología}

Estudio de tipo Descriptivo Correlacional. El Universo considerado la población de la ciudad de Neiva en edades entre 18 y 75 años, la cual se estimó en 197.207 sujetos (91.736 hombres y 105.471 mujeres) (DANE, 2009). El tamaño de la muestra es representativo y para su cálculo se siguieron los siguientes parámetros poblacionales: Nivel de confianza del 95\%; con igual probabilidad de selección; y Error relativo del 5\%. Se realizó un muestreo aleatorio simple en la selección de la muestra de acuerdo al interés por la convocatoria de participar en el programa "Neiva Unida y Saludable" y estuvo conformada por 972 sujetos de la ciudad de Neiva, 470 mujeres que representan el 48,4\% y 502 hombres el 51,6\% en el rango de (Mujeres con un intervalo de 45,2 $\pm 15,2$ años y los hombres de $44,4 \pm 14,8$ años para la media). La muestra fue suficientemente representativa garantizando la normalidad para efectos de las estimaciones que se realizaron en el modelo.

En cuanto a la recolección de los datos, después de explicar a los sujetos los objetivos y procedimientos generales del estudio, firmaron la hoja de consentimiento informado (Minsalud, 1993; WMA, 2014). Se aplicó una encuesta estructurada con el fin de obtener los hábitos de actividad física, clasificándolos de la siguiente manera: Inactivos (No realizan Actividad Física); Activos (Practica Actividad Física 3 o más veces por semana, 20min o más). El sujeto al llegar a la Valoración y durante el proceso de diligenciamiento de la Ficha de Registro se le puso el Monitor de Ritmo Cardiaco (Polar F11 o Polar - E.U.) FS1 con el cual permanecía durante 10min en posición sentado, relajado, tranquilo y se tomaba la FCR, al igual que la TAS y TAD, y con el fin de evitar los errores sistemáticos o sesgos del observador y de agilizar la toma de datos, se utilizó un equipo electrónico semiautomático (OMRON 705CP - E.U.) (Artigao et al., 2000; Divison, Escobar \& Segui, 2015).

Para la valoración de los niveles de adiposidad, se utilizaron las técnicas de medición antropométrica recomendadas por la ISAK (Marfell-Jones, Olds, Stewart \& Carter, 2006), registrando un total de 20 medidas, entre ellas el Peso Corporal (Báscula SECA 700), Estatura (Estadiómetro portátil WCS-WOOD-Brasil), pliegues cutáneos (Plicómetro CESCORF-Brasil), Diámetros (Calibrador FAGA-S.R.L-Brasil) y Perímetros (Cinta Antropométrica modelo WISO-R88-Brasil). Se calculó el IMC creado por Adolph Quetelet (1.796 - 1.874) (Stewart, 2010); los parámetros de sobrepeso y obesidad se establecieron de acuerdo a la Clasificación Internacional de la población adulta de peso insuficiente, sobrepeso y obesidad (WHO, 2006); el ICC, el cual es un índice asociado a la grasa visceral y la grasa intraabdominal, se realizó una prueba sencilla que mide la razón circunferencia de la cintura/cadera, estimándose factor de riesgo alto para los hombres un ICC $\geq 1,0$ y mujeres $\geq 0,85$ (Morikawa et al., 2011); para determinar el \%GC, se aplicó la Formula de Yuhasz para sujetos sedentarios mayores de 18 años (Yuhasz, 1977; Ordaz, 2006; Leyk et al., 2006), así: 
\%GC Mujeres $=((\operatorname{Tri}+\mathrm{Su}+\mathrm{Si}+\mathrm{Abd}+\mathrm{M}+\mathrm{Pa}) * 0,1429)+4.56$

$\%$ GC Hombres $=(($ Tri $+\mathrm{Su}+\mathrm{Si}+\mathrm{Abd}+\mathrm{M}+\mathrm{Pa}) * 0,097)+3.64$

Donde: Tríceps: Tri

Abdominal: Abd
Subescapular: Su

Muslo: M
Suprailiaco: Si

Pantorrilla: $\mathrm{Pa}$

Para la valoración de la aptitud cardiorrespiratoria se aplicó el Test de Rockport - test de la milla (Dolgener, Hensley, Marsh \& Fjelstul, 1994; Fenstermaker, Plowman \& Looney, 1992), el cual ha sido descrito y validado en varias publicaciones para estimar el $\mathrm{VO}_{2}$ máx, aplicando la siguiente ecuación:

VO 2 máx $=132,853-[0,1692 x P]-[0,3877 x E)]+[6,3150 x G]-[3,2649 x T)]-[0,1565 x F C]$

Donde: $\mathrm{P}=$ Peso corporal en libras

Genero (0 = Femenino 1 = Masculino $)$
$\mathrm{E}=$ Edad en años

$\mathrm{T}=$ Tiempo de realización de la prueba (minutos)

FC = Frecuencia cardiaca al final de la prueba (lat $/ \mathrm{min}$.

El análisis de datos de la presente investigación partió de la elaboración de una base de datos que agrupó las referencias de cada uno de los sujetos. Se construyó una base de datos, para este estudio se utilizaron los paquetes estadísticos SPSS Versión 23 e InfoStat/Profesional versión 1,2. La metodología estadística inicial partió de la descripción de las variables en estudio, posteriormente se determinó el grado de asociación entre las variables estudiadas con una matriz de correlaciones (coeficiente de correlación de Pearson). Para comparar los grupos etareos, se optó por una prueba ANOVA y luego una comparación multiple LSD Fisher, con el fin de observar los mejores comportamientos en cada una de las variables dentro del grupo de sujetos inactivos y activos; por último, se estableció el grado de agrupamiento entre las variables en estudio a partir del análisis de conglomerados jerárquico, utilizando la técnica de agrupamiento encadenamiento promedio (Average Linkage).

\section{Resultados}

Se evaluaron 972 sujetos de la ciudad de Neiva - Colombia, 470 mujeres que representan el 48,4\% y 502 hombres el 51,6\% entre los 18 y 75 años de edad (Mujeres con un intervalo de 45,2 $\pm 15,2$ años y los hombres con un intervalo de 44,4 $\pm 14,8$ años para la media). Uno de los resultados más destacables es que el nivel de inactividad es considerablemente mayor en los hombres que en las mujeres, así, el 36,8\% de los hombres es Activo y el 63,1\% Inactivo, mientras que las mujeres el 67,9\% Activas, frente al 32,1\% que son Inactivas, mientras al considerar la edad, los más jóvenes de la población analizada (18 a 24 años), fueron los que presentaron los mayores niveles de inactividad física, el 71,9\% de los hombres y el 43,1\% de las mujeres, tal como se aprecia en la Tabla 1.

Tabla 1. Distribución porcentual de Niveles de Actividad Física según género y grupo etareo

\begin{tabular}{|c|c|c|c|c|c|c|c|c|c|c|c|c|c|c|c|}
\hline \multirow[t]{2}{*}{ Género } & \multirow{2}{*}{$\begin{array}{l}\text { Nivel De } \\
\text { Actividad } \\
\text { Física }\end{array}$} & \multicolumn{2}{|c|}{$\begin{array}{c}18 \text { a } 24 \\
\text { Años }\end{array}$} & \multicolumn{2}{|c|}{$\begin{array}{c}25 \text { a } 34 \\
\text { Años }\end{array}$} & \multicolumn{2}{|c|}{$\begin{array}{c}35 \text { a } 44 \\
\text { Años }\end{array}$} & \multicolumn{2}{|c|}{$\begin{array}{c}45 \text { a } 54 \\
\text { Años }\end{array}$} & \multicolumn{2}{|c|}{$\begin{array}{c}55 \text { a } 64 \\
\text { Años }\end{array}$} & \multicolumn{2}{|c|}{$\begin{array}{c}\geq \mathbf{6 5} \\
\text { Años }\end{array}$} & \multicolumn{2}{|c|}{$\begin{array}{c}\text { Total por } \\
\text { Género }\end{array}$} \\
\hline & & $\mathbf{N}$ & $\%$ & $\mathbf{N}$ & $\%$ & $\mathbf{N}$ & $\%$ & $\mathbf{N}$ & $\%$ & $\mathbf{N}$ & $\%$ & $\mathbf{N}$ & $\%$ & $\mathbf{N}$ & $\%$ \\
\hline \multirow{3}{*}{ Hombres } & Inactivo & 46 & 71,9 & 50 & 61,7 & 55 & 59,8 & 84 & 68,3 & 64 & 60,4 & 18 & 50 & 317 & 63,1 \\
\hline & Activo & 18 & 28,1 & 31 & 38,3 & 37 & 40,2 & 39 & 31,7 & 42 & 39,6 & 18 & 50 & 185 & 36,8 \\
\hline & Total & 64 & 100 & 81 & 100 & 92 & 100 & 123 & 100 & 106 & 100 & 36 & 100 & 502 & 100 \\
\hline \multirow{3}{*}{ Mujeres } & Inactivo & 28 & 43,1 & 27 & 42,2 & 23 & 29,5 & 33 & 29,7 & 30 & 27,5 & 10 & 23,3 & 151 & 32,1 \\
\hline & Activo & 37 & 56,9 & 37 & 57,8 & 55 & 70,5 & 78 & 70,3 & 79 & 72,5 & 33 & 76,7 & 319 & 67,9 \\
\hline & Total & 65 & 100 & 64 & 100 & 78 & 100 & 111 & 100 & 109 & 100 & 43 & 100 & 470 & 100 \\
\hline
\end{tabular}


Al observar el comportamiento de las variables estudiadas según género (Prueba T para muestras independientes según género asumiendo varianzas iguales), se evidenciaron diferencias significativas entre hombres y mujeres en las variables ICC y FCR (Tabla 2).

Tabla 2. Prueba T para muestras independientes asumiendo varianzas iguales según género

\begin{tabular}{|l|c|c|c|c|c|}
\hline \multirow{2}{*}{ Variable } & \multicolumn{2}{|c|}{$\begin{array}{c}\text { Prueba de Levene para } \\
\text { la igualdad de varianzas }\end{array}$} & \multicolumn{2}{|c|}{ Prueba T para la igualdad de medias } \\
\cline { 2 - 6 } & $\mathbf{F}$ & Sig. & $\mathbf{t}$ & $\mathbf{g l}$ & Sig. (bilateral) \\
\hline IMC $\left(\mathrm{kg} \cdot \mathrm{m}^{-2}\right)$ & 2,04 & 0,15 & $-0,95$ & 970,00 & 0,34 \\
\hline ICC & $\mathbf{1 , 4 3}$ & $\mathbf{0 , 2 3}$ & $-\mathbf{- 2 , 2 6}$ & $\mathbf{9 7 0 , 0 0}$ & $\mathbf{0 , 0 2}$ \\
\hline \%GC & 0,40 & 0,53 & $-0,37$ & 970,00 & 0,71 \\
\hline FCR (lat/min) & $\mathbf{0 , 0 0}$ & $\mathbf{0 , 9 5}$ & $-\mathbf{2 , 1 4}$ & $\mathbf{9 7 0 , 0 0}$ & $\mathbf{0 , 0 3}$ \\
\hline TAS $(\mathrm{mmHg})$ & 0,75 & 0,39 & 1,42 & 970,00 & 0,16 \\
\hline TAD $(\mathrm{mmHg})$ & 0,02 & 0,88 & 1,21 & 970,00 & 0,23 \\
\hline $\mathrm{VO}_{2} \mathrm{máx}\left(\mathrm{ml} \cdot \mathrm{kg}^{-1} \cdot \mathrm{min}^{-1}\right)$ & 0,57 & 0,45 & 0,58 & 970,00 & 0,56 \\
\hline
\end{tabular}

Mientras éste mismo procedimiento de acuerdo a los niveles de actividad física (inactivos y activos) arrojó diferencias significativas en el IMC, la FCR y el $\mathrm{VO}_{2}$ máx como lo indica la Tabla 3

Tabla 3. Prueba T para muestras independientes asumiendo varianzas iguales según nivel de actividad física

\begin{tabular}{|l|c|c|c|c|c|}
\hline \multirow{2}{*}{ Variable } & \multicolumn{2}{|c|}{$\begin{array}{c}\text { Prueba de Levene } \\
\text { para la igualdad de } \\
\text { varianzas }\end{array}$} & \multicolumn{2}{|c|}{ Prueba T para la igualdad de medias } \\
\cline { 2 - 6 } & $\mathbf{F}$ & Sig. & $\mathbf{t}$ & gl & $\begin{array}{c}\text { Sig. } \\
\text { (bilateral) }\end{array}$ \\
\hline IMC (kg·m-2 $)$ & $\mathbf{1 0 , 5 3}$ & $\mathbf{0 , 0 0}$ & $-\mathbf{3 , 1 7}$ & $\mathbf{9 7 0 , 0 0}$ & $\mathbf{0 , 0 0}$ \\
\hline ICC & 1,20 & 0,27 & 0,93 & 970,00 & 0,35 \\
\hline \%GC & 3,99 & 0,05 & $-0,67$ & 970,00 & 0,50 \\
\hline FCR (lat $/ \mathbf{m i n})$ & $\mathbf{3 , 7 7}$ & $\mathbf{0 , 0 5}$ & $-\mathbf{2 , 9 9}$ & $\mathbf{9 7 0 , 0 0}$ & $\mathbf{0 , 0 0}$ \\
\hline TAS (mmHg) & 0,94 & 0,33 & $-1,15$ & 970,00 & 0,25 \\
\hline TAD (mmHg) & 0,03 & 0,87 & $-1,23$ & 970,00 & 0,22 \\
\hline VO $_{2} \mathbf{m a ́ x ~}\left(\mathbf{m l} \cdot \mathbf{k g}^{-1} \cdot \mathbf{m i n}^{-1}\right.$ ) & $\mathbf{1 , 0 1}$ & $\mathbf{0 , 3 1}$ & $\mathbf{2 , 2 7}$ & $\mathbf{9 7 0 , 0 0}$ & $\mathbf{0 , 0 2}$ \\
\hline
\end{tabular}

El estudio permitió arrojar grados de asociación (coeficiente de correlación de Pearson) menores del 5\%, lo cual rechaza la hipótesis de independencia entre las siguientes variables: el IMC se asocia con ICC, \%GC, FCR y el $\mathrm{VO}_{2}$ máx; el ICC mostró asociación con el \%GC, TAS, TAD y el VO máx; el \%GC con FCR, la TAD y el $\mathrm{VO}_{2}$ máx; la FCR con el $\mathrm{VO}_{2}$ máx; y por último la TAS con la TAD y el VO 2 máx (Tabla 4). 
Tabla 4. Matriz de correlación/Probabilidades

\begin{tabular}{|c|c|c|c|c|c|c|c|}
\hline Variable & IMC & ICC & \%GC & FCR & TAS & TAD & VO $_{2}$ máx \\
\hline IMC & & & & & & & \\
\hline ICC & $\mathbf{0 , 0 0}$ & & & & & & \\
\hline \%GC & $\mathbf{0 , 0 0}$ & $\mathbf{0 , 0 5}$ & & & & & \\
\hline FCR & $\mathbf{0 , 0 0}$ & 0,07 & $<\mathbf{0 , 0 0 0 1}$ & & & & \\
\hline TAS & 0,94 & $\mathbf{0 , 0 2}$ & 0,28 & 0,26 & & & \\
\hline TAD & 0,90 & $\mathbf{0 , 0 0}$ & $\mathbf{0 , 0 4}$ & 0,97 & $<\mathbf{0 , 0 0 0 1}$ & & \\
\hline VO $_{2}$ máx & $<\mathbf{0 , 0 0 0 1}$ & $<\mathbf{0 , 0 0 0 1}$ & $<\mathbf{0 , 0 0 0 1}$ & $<\mathbf{0 , 0 0 0 1}$ & $\mathbf{0 , 0 0}$ & 0,42 & \\
\hline
\end{tabular}

Con el fin de observar el comportamiento de las variables objeto de estudio se optó por el pocedimiento ANOVA de un factor intergrupos - grupo etareo- según el nivel de actividad física, que evidenció diferencias significativas en las variables IMC, FCR y TAS en los sujetos inactivos; mientras en los activos dichas diferencias se presentaron en las variables IMC, FCR y $\mathrm{VO}_{2}$ máx (Tabla 5).

Tabla 5. Tabla resumen del procedimiento ANOVA de un factor intergrupos -grupo etareo- según nivel de actividad física

\begin{tabular}{|l|c|c|c|c|}
\hline \multirow{2}{*}{ Variable } & \multicolumn{2}{|c|}{ Inactivos } & \multicolumn{2}{c|}{ Activos } \\
\cline { 2 - 5 } & $\mathbf{F}$ & Sig. & F & Sig. \\
\hline IMC $\left(\mathrm{kg} \cdot \mathrm{m}^{-2}\right)$ & $\mathbf{7 , 0 0}$ & $\mathbf{0 , 0 0}$ & $\mathbf{7 , 1 6}$ & $\mathbf{0 , 0 0}$ \\
\hline ICC & 0,75 & 0,58 & 1,47 & 0,20 \\
\hline \%GC & 1,62 & 0,15 & 1,54 & 0,18 \\
\hline FCR $(\mathrm{lat} / \mathrm{min})$ & $\mathbf{4 , 3 8}$ & $\mathbf{0 , 0 0}$ & $\mathbf{2 , 6 6}$ & $\mathbf{0 , 0 2}$ \\
\hline TAS $(\mathrm{mmHg})$ & $\mathbf{3 , 3 5}$ & $\mathbf{0 , 0 1}$ & 1,77 & 0,12 \\
\hline TAD $(\mathrm{mmHg})$ & 0,80 & 0,55 & 1,59 & 0,16 \\
\hline VO $_{2} \mathbf{m a ́ x}\left(\mathrm{ml} \cdot \mathrm{kg}^{-1} \cdot \mathrm{min}^{-1}\right)$ & 0,87 & 0,50 & $\mathbf{2 , 8 9}$ & $\mathbf{0 , 0 5}$ \\
\hline
\end{tabular}

Para determinar de manera específica en que grupos de edades se presentaron los mejores resultados y en cuales de ellos se evidenciaron diferencias significativas, se implementó la prueba de comparación múltiple LSD Fisher la cual determinó que: a) El grupo de sujetos inactivos mostró diferencias significativas en las variables IMC, FCR y TAS; los valores más bajos del IMC $\left(24,59 \mathrm{~kg} \cdot \mathrm{m}^{-2}\right)$ y la TAS $(109,93 \mathrm{mmHg})$ los arrojó el grupo de 18 a 24 años de edad; y los más altos, los sujetos $\geq 65$ años (IMC 28,96kg $\cdot \mathrm{m}^{-2}$ y TAS 118,61mmHg); mientras la FCR más baja la obtuvieron los sujetos $\geq 65$ años (72,71lat/min) y la más alta los de 18 a 24 años (81,04lat/min); b) El grupo de sujetos activos, arrojó diferencias significativas en el IMC, FCR y $\mathrm{VO}_{2}$ máx; los sujetos de 18 a 24 años de edad mostraron el IMC más bajo $\left(25,09 \mathrm{~kg} \cdot \mathrm{m}^{-2}\right)$ y los $\geq 65$ años el más alto $\left(31,36 \mathrm{~kg} \cdot \mathrm{m}^{-2}\right)$; la FCR más baja la obtuvo el grupo de 55 a 64 años de edad (75,19 lat/min) y la más alta los sujetos $\geq 65$ años (81,06 lat/min); en lo que se refiere al $\mathrm{VO}_{2}$ máx, los sujetos de 35 a 44 años arrojaron el valor más bajo $\left(30,16 \mathrm{ml} \cdot \mathrm{kg}^{-1} \cdot \mathrm{min}^{-1}\right)$, mientras el más alto fue para los de 55 a 64 años de edad $\left(35,75 \mathrm{ml} \cdot \mathrm{kg}^{-1} \cdot \mathrm{min}^{-1}\right)$. (Tabla 6). 
Tabla 6. Prueba de comparación múltiple LSD Fisher Alfa= 0,05 - Grupos de edades según nivel de actividad física

\begin{tabular}{|c|c|c|c|c|c|c|c|c|c|c|c|c|c|c|}
\hline \multicolumn{7}{|c|}{ INACTIVOS } & \multicolumn{8}{|c|}{ ACTIVOS } \\
\hline Variable & $\begin{array}{l}\text { Grupo } \\
\text { Etareo }\end{array}$ & Medias & $\mathbf{n}$ & & & & Variable & $\begin{array}{l}\text { Grupo } \\
\text { Etareo }\end{array}$ & Medias & $\mathbf{n}$ & & & & \\
\hline IMC & $18-24$ & 24,59 & 74 & A & & & IMC & $18-24$ & 25,09 & 55 & A & & & \\
\hline & $35-44$ & 25,95 & 78 & A & & & & $25-34$ & 27,39 & 68 & & B & & \\
\hline & $25-34$ & 27,85 & 77 & & B & & & $45-54$ & 27,86 & 117 & & B & C & \\
\hline & $45-54$ & 28,20 & 117 & & B & & & $35-44$ & 29,15 & 92 & & B & C & \\
\hline & $55-64$ & 28,21 & 94 & & B & & & $55-64$ & 29,29 & 121 & & & $\mathrm{C}$ & \\
\hline & $\geq 65$ & 28,96 & 28 & & B & & & $\geq 65$ & 31,36 & 51 & & & & D \\
\hline FCR & $\geq 65$ & 72,71 & 28 & A & & & FCR & $55-64$ & 75,19 & 121 & A & & & \\
\hline & $35-44$ & 73,33 & 78 & A & & & & $45-54$ & 76,83 & 117 & A & B & & \\
\hline & $45-54$ & 74,66 & 117 & A & & & & $35-44$ & 78,68 & 92 & & B & $\mathrm{C}$ & \\
\hline & $55-64$ & 75,00 & 94 & A & & & & $25-34$ & 79,65 & 68 & & B & C & \\
\hline & $25-34$ & 75,92 & 77 & A & & & & $18-24$ & 80,53 & 55 & & B & C & \\
\hline & $18-24$ & 81,04 & 74 & & B & & & $\geq 65$ & 81,06 & 51 & & & $\mathrm{C}$ & \\
\hline TAS & $18-24$ & 109,93 & 74 & A & & & $\mathrm{VO}_{2}$ máx & $35-44$ & 30,16 & 92 & A & & & \\
\hline & $45-54$ & 110,60 & 117 & A & & & & $18-24$ & 31,53 & 55 & A & B & & \\
\hline & $55-64$ & 110,80 & 94 & A & B & & & $\geq 65$ & 31,92 & 51 & A & B & & \\
\hline & $25-34$ & 114,88 & 77 & & B & C & & $45-54$ & 32,98 & 117 & A & B & & \\
\hline & $35-44$ & 115,01 & 78 & & & C & & $25-34$ & 33,58 & 68 & A & B & & \\
\hline & $\geq 65$ & 118,61 & 28 & & & $\mathrm{C}$ & & $55-64$ & 35,75 & 121 & & B & & \\
\hline
\end{tabular}

Letras distintas indican diferencias significativas ( $\mathrm{p} \leq 0,05)$, las letras A, B y C representan los grupos en estudio de acuerdo al interés de la investigación.

Al comparar los resultados de las variables los grupos de edades según género, se evidenció que tanto hombres como mujeres presentaron diferencias estadísticamente significativas entre al menos dos medias con una confiabilidad del 95,0\% en las variables IMC, FCR, TAS y TAD.

Por último, y con el fin de determinar el agrupamiento natural de las variables estudiadas y el grado de dicha asociación, en primera instancia se realizó un análisis de conglomerados jerárquico, utilizando el método encadenamiento promedio (Average Linkage) y una distancia Euclídiana para establecer el número de conglomerados, fijando un criterio de corte en una distancia del 50\% $(1079,38)$ de la distancia máxima existente en los datos (variables) y el contenido de los mismos (Figura 1). 
Figura 1. Dendrograma usando el Método Promedio (Average linkage) entre variables

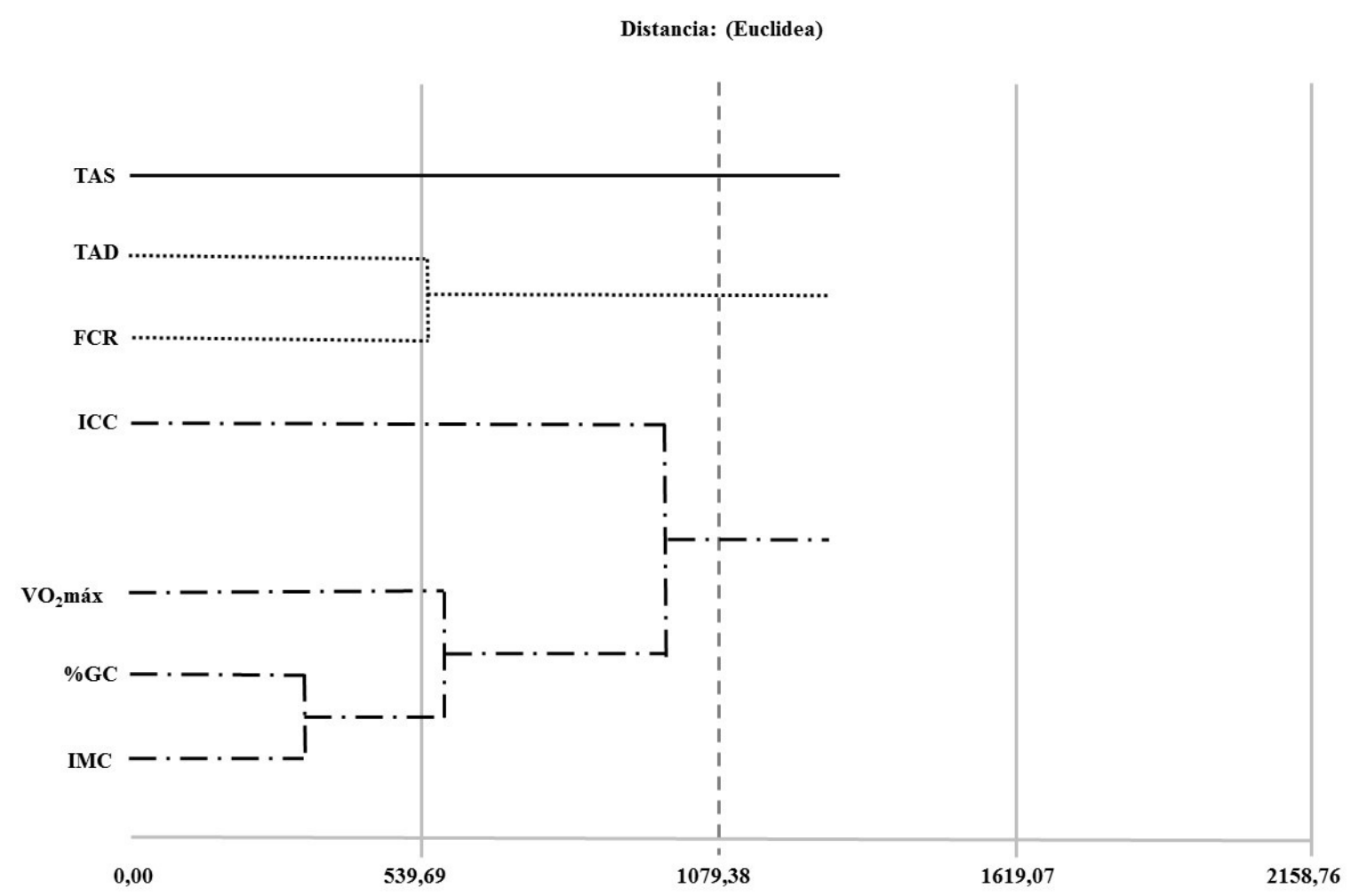

El dendrograma sugiere que la mejor solución es la de tres conglomerados, un primer grupo formado por las variables IMC $\left(\mathrm{kg} \cdot \mathrm{m}^{-2}\right)$, ICC, \%GC y VO $\mathrm{máx}\left(\mathrm{ml} \cdot \mathrm{kg}^{-1} \cdot \mathrm{min}^{-1}\right)$; un segundo grupo con las variables FCR (lat/min) y TAD (mmHg); y el grupo tres, la variable TAS (mmHg).

\section{Discusión}

Los niveles de práctica de actividad física de los sujetos evaluados son preocupantes, ya que se observaron prevalencias bajas en el cumplimiento de las recomendaciones para dicha práctica (Haskell et al., 2007), siendo altos los niveles de inactividad física encontrados, los cuales se convierten en factores modificables de riesgo de enfermedad cardiovascular importantes (Thompson et al., 2003) y que se asocian con un mayor riesgo de eventos cardiovasculares fatales y no fatales en hombre como en mujeres (Hu et al., 2005; Manson et al., 2002).

$\mathrm{Al}$ analizar el nivel de actividad física en relación al género y la edad, se observa que el género femenino presenta los porcentajes más altos de práctica de Actividad física (67,87\%) frente a un 36,85\% de los hombres, lo cual contrasta con los resultados de diversos estudios que muestran los niveles más altos de actividad física en la población masculina (González et al., 2014).

Partiendo del hecho que la Aptitud Cardiorrespiratoria es una medida objetiva que refleja las influencias funcionales de los hábitos de actividad física, la genética y el estado de enfermedad, se observó que la muestra no presentó diferencias significativas del $\mathrm{VO}_{2}$ máx al comparar los hombres y las mujeres, sin embargo, el género masculino presentó los mayores valores, con un intervalo para la media de $34,2 \pm 14,1 \mathrm{ml} \cdot \mathrm{kg}^{-1} \cdot \mathrm{min}^{-1}$, mientras las mujeres $33,6 \pm 13,5 \mathrm{ml} \cdot \mathrm{kg}^{-1} \cdot \mathrm{min}^{-1}$, valores mucho menores a los encontrados en la versión original de la prueba de Rockport, realizada en hombres y mujeres entre los 30 y 69 años de edad (Kline, Porcari \& Hintermeister, 1987), en el que se encontró un intervalo para la media de $41,2 \pm 8,09 \mathrm{ml} \cdot \mathrm{kg}^{-1} \cdot \mathrm{min}^{-1}$, aunque sí se apreciaron diferencias significativas al comparar sujetos Activos con Inactivos. 
De igual forma, en los datos obtenidos, no se aprecia una correlación entre la edad y los niveles de $\mathrm{VO}_{2}$ máx. Resaltando que los valores encontrados son superiores a los arrojados durante la aplicación de la prueba en poblaciones de las mismas características de edad y género $\left(\approx 25,5 \mathrm{ml} \cdot \mathrm{kg}^{-1} \cdot \mathrm{min}^{-1}\right)$ (Fenstermaker, Plowman \& Looney, 1992).

En los parámetros de adiposidad, los resultados del IMC del total de la muestra arrojó un intervalo para la media de $27,85 \pm 5,85 \mathrm{~kg} / \mathrm{m}^{2}$, valor clasificado como sobrepeso, de acuerdo a la OMS (WHO, 2004), siendo una voz de alerta, ya que el sobrepeso junto con la inactividad física están asociadas con la mortalidad prematura, mayor riesgo de morbilidad de enfermedades crónicas y discapacidades funcionales (Kwon et al., 2015; Trivedi et al., 2015) e identificados como los principales factores de riesgo modificables para la enfermedad cardíaca coronaria (Blair \& Brodney, 1999; Ecclestone, Jones \& WHO, 2004). Al considerar éste mismo factor, de acuerdo al género, no se presentaron diferencias significativas entre hombres y mujeres $(\mathrm{p}=0,322)$, mientras al correlacionarlo con el nivel de actividad física, se evidenciaron dichas diferencias entre ellos con un valor de $\mathrm{p}=0,002$, siendo los valores más altos los registrados en el grupo de inactivos con respecto al grupo de activos, lo cual coincide con lo resultados de los estudios consultados (Malmberg, Miilunpalo, Pasanen, Vuori \& Oja, 2005; Johnson, Wu, Bonow \& Holly, 2008; Hu, Tuomilehto, Silventoinen, Barengo \& Jousilahti, 2004).

El \%GC, en las mujeres fue de 22,39 \pm 6 , considerándose dentro de un valor promedio para ellas, mientras que para hombres fue menor 22,31 \pm 6 , siendo un indicador de sobrepeso (Paniagua, Lohsoonthorn, Lertmaharit, Jiamjarasrangsi \& Williams, 2008); el ICC, no evidenció diferencias significativas al correlacionarlo con la actividad física, subrayando, que los niveles de actividad física entre la población objeto de estudio son poco activos, es probable que esta sea la explicación de que no existan esas diferencias, mientras, al realizar dicha correlación entre géneros, las diferencias fueron significativas, siendo el grupo femenino el que obtuvo los valores más altos, prácticamente en todos los grupos de edad, así: El grupo de 25 a 34 años de edad con 0,86 $\pm 0,17$; los de 45 a 54 y de 55 a 64 años de edad con 0,85 $\pm 0,1$; siendo un parámetro de riesgo alto de enfermedad cardiovascular (Hu, Tuomilehto, Silventoinen, Barengo \& Jousilahti, 2004; Janssen, Katzmarzyk \& Ross, 2005; Sui et al., 2007); de acuerdo a la edad y género, los valores más altos del \%GC se presentaron en el género femenino en las $\geq 65$ años con 23,59 $\pm 6,4$ seguidas por las de 18 a 24 años de edad con 23,52 $\pm 6,3$; en los hombres al igual que en las mujeres los valores más altos está en los $\geq 65$ años con 23,46 $\pm 7,0$ seguidos por los de 25 a 34 años con 22,8 $\pm 6,8$ caracterizándose por un aumento asistemático del \%GC con la edad, lo cual difiere de los estudios consultados, en los cuales se han encontrado aumentos de la grasa corporal con la edad entre los 18 y 79 años (Wu et al., 2007; Mott et al., 1999), en la muestra se observa que en los grupos más jóvenes se encontraron los mayores porcentajes de inactividad, tal vez este parámetro explique las diferencias en los niveles de grasa corporal con respecto a los estudios consultados que no tenían en cuenta la actividad física, ya que la práctica moderada de ella puede influir en el \%GC (Osei-Tutu \& Campagna, 2005).

Considerando el comportamiento de las variables de adiposidad y aptitud cardiorrespiratoria según el nivel de actividad física (inactivo y activo) de la muestra evaluada, se evidenciaron diferencias significativas en el IMC, la FCR y el $\mathrm{VO}_{2}$ máx, siendo datos preocupantes de riesgo cardiovascular en la población inactiva (Pahor et al., 2006; Osei-Tutu \& Campagna, 2005; Thompson et al., 2003).

Un punto relevante en el presente estudio, es que permitió arrojar grados de asociación con un nivel de significancia del 5\%, entre las variables correspondientes a la Aptitud Cardiorrespiratoria con las de Adiposidad, lo cual rechaza la hipótesis de independencia entre dichos factores, así: El IMC con el ICC, el \%GC, la FCR y el $\mathrm{VO}_{2}$ máx; el ICC mostró relación con el \%GC, la TAS, la TAD y el $\mathrm{VO}_{2}$ máx; el \%GC con la FCR, la TAD y el $\mathrm{VO}_{2}$ máx; la FCR evidenció correlación con el $\mathrm{VO}_{2}$ máx; y por último, la TAS con la TAD y el $\mathrm{VO}_{2}$ máx, lo cual coincide con diferentes estudios donde adultos con sobrepeso y obesos que logran niveles adecuados de actividad física, y por ende, mejores parámetros de su aptitud cardiorrespirtaria, presentan un menor riesgo de morbilidad y mortalidad (Mott et al., 1999). Del mismo modo, al realizar una comparación múltiple (LSD Fisher), los datos son preocupantes, pues como se ha mencionado, la prevalencia de sobrepeso, obesidad y sedentarismo ha aumentado a 
nivel local, regional como nacional durante las últimas décadas (Ecclestone, Jones \& WHO, 2004).

De acuerdo a las variables estudiadas y el tipo de estudio, se sugiere una fuerte asociación de las variables en tres conglomerados, fijando como criterio de corte una distancia del 50\% $(1079,38)$ de la distancia máxima existente en las variables estudiadas y el contenido de las mismas, corroborando nuevamente el mayor coeficiente de correlación de las variables que hacen parte de cada conglomerado, así: un primer grupo formado por las variables IMC $\left(\mathrm{kg} \cdot \mathrm{m}^{-2}\right)$, ICC, \%GC y VO 2 máx $\left(\mathrm{ml} \cdot \mathrm{kg}^{-1} \cdot \mathrm{min}^{-1}\right)$; un segundo grupo con las variables FCR (lat/min) y TAD (mmHg); y el grupo tres, la variable TAS (mmHg).

\section{Conclusiones}

El comportamiento entre mujeres y hombres de la muestra analizada difiere con relación a los niveles de actividad física, encontrándose diferencias significativas a favor de las mujeres poco activas frente a los hombres, donde en éstos últimos prevalece la inactividad física. De acuerdo a los resultados de los parámetros de adiposidad (IMC, ICC y \%GC) los valores más altos de cada uno de los parámetros se presentaron en el género femenino, quienes se caracterizaron por los índices de sobrepeso y obesidad, mientras los de aptitud cardiorrespiratoria, como el $\mathrm{VO}_{2}$ máx estimado, fue mayor en los hombres.

El estudio evidenció diferencias significativas en el IMC, la FCR y el $\mathrm{VO}_{2}$ máx según el nivel de actividad física (inactivos y activos). Al igual, una asociación con nivel de significancia del 5\%, entre el IMC con ICC, \%GC, FCR y el $\mathrm{VO}_{2}$ máx; el ICC con el \%GC, TAS, TAD y el $\mathrm{VO}_{2}$ máx; el \%GC con FCR, la TAD y el $\mathrm{VO}_{2}$ máx; la FCR con el $\mathrm{VO}_{2}$ máx; y por último la TAS con la TAD y el $\mathrm{VO}_{2}$ máx lo cual rechaza la hipótesis de independencia.

Los grupos de edades de los sujetos inactivos evidenciaron diferencias significativas en las variables IMC, FCR y TAS; mientras en los activos dichas diferencias se presentaron en las variables IMC, FCR y VO 2 máx; al comparar la muestra por género, dichas diferencias se presentaron entre al menos dos medias con una confiabilidad del 95,0\% en las variables IMC, FCR, TAS y TAD.

Como conclusión general, los resultados obtenidos de este estudio, revelan de manera clara, una población potencialmente en riesgo, ya que los indicadores antropométricos de adiposidad (IMC, \%GC y el ICC), y la valoración de la aptitud cardiorrespiratoria $\left(\mathrm{VO}_{2}\right.$ máx.) ponen de manifiesto una combinación de factores de riesgo para la presencia de enfermedades cardiovasculares no transmisibles.

\section{Referencias}

ACSM. (1993). American College of Sports Medicine. Position Stand. Physical activity, physical fitness, and hypertension. Med Sci Sports Exerc, 25(10), i-x.

ACSM. (1995). American College of Sports Medicine position stand. Osteoporosis and exercise. Med Sci Sports Exerc, 27(4), i-vii.

Artigao, L. M., Llavador, J. J., Puras, A., Lopez Abril, J., Rubio, M. M., Torres, C.,...Fuentes, G. (2000). [Evaluation and validation of Omron Hem 705 CP and Hem 706/711 monitors for self-measurement of blood pressure]. Aten Primaria, 25(2), 96-102.

Blacklock, R. E., Rhodes, R. E., y Brown, S. G. (2007). Relationship between regular walking, physical activity, and health-related quality of life. J Phys Act Health, 4(2), 138-152.

Blair, S. N., y Brodney, S. (1999). Effects of physical inactivity and obesity on morbidity and mortality: current evidence and research issues. Med Sci Sports Exerc, 31(11 Suppl), S646-662. 
Buchheit, M., Simon, C., Charloux, A., Doutreleau, S., Piquard, F., y Brandenberger, G. (2006). Relationship between very high physical activity energy expenditure, heart rate variability and self-estimate of health status in middle-aged individuals. Int J Sports Med, 27(9), 697-701. doi: https://doi.org/10.1055/s-2005-872929

DANE. (2009). Estudios Postcensales No. 7. Proyecciones nacionales y departamentales de población 2005 2020. http://www.dane.gov.co/files/investigaciones/poblacion/proyepobla06 20/7Proyecciones poblacion.pdf

Després, J. P., Pouliot, M. C., Moorjani, S., Nadeau, A., Tremblay, A., Lupien, P. J.,...Bouchard, C. (1991). Loss of abdominal fat and metabolic response to exercise training in obese women. Am J Physiol, 261(2 Pt 1), E159167.

Divison Garrote, J. A., Escobar Cervantes, C., y Segui Diaz, M. (2015). [Blood pressure and incidence of 12 cardiovascular diseases: Risks throughout life, life years lost and relationship between different age groups in 1.25 nillion people]. Semergen, 41(1), 50-51. doi: https://doi.org/10.1016/j.semerg.2014.07.014

Dolgener, F. A., Hensley, L. D., Marsh, J. J., y Fjelstul, J. K. (1994). Validation of the Rockport Fitness Walking Test in college males and females. Res Q Exerc Sport, 65(2), 152-158. doi: https://doi.org/10.1080/02701367.1994.10607610

Ecclestone, N. A., Jones, J., y WHO. (2004). International Curriculum Guidelines for Preparing Physical Activity Instructors of Older Adults, in collaboration with the Aging and Life Course, World Health Organization. J Aging Phys Act, 12(4), 467-479.

Fenstermaker, K. L., Plowman, S. A., y Looney, M. A. (1992). Validation of the Rockport Fitness Walking Test in females 65 years and older. Res Q Exerc Sport, 63(3), 322-327. doi: https://doi.org/10.1080/02701367.1992.10608749

González, S. A., Sarmiento, O. L., Cohen, D. D., Camargo, D. M., Correa, J. E., Páez, D. C.,...Ramírez-Vélez, R. (2014). Results from Colombia's 2014 Report Card on Physical Activity for Children and Youth. J Phys Act Health, 11 Suppl 1, S33-44. doi: https://doi.org/10.1123/jpah.2014-0170

Haskell, W. L., Lee, I. M., Pate, R. R., Powell, K. E., Blair, S. N., y Franklin, B. A. (2007). Physical activity and public health: updated recommendation for adults from the American College of Sports Medicine and the American Heart Association. Circulation, 116(9), 1081-1093. doi: https://doi.org/10.1161/CIRCULATIONAHA.107.185649

Hu, G., Sarti, C., Jousilahti, P., Silventoinen, K., Barengo, N. C., y Tuomilehto, J. (2005). Leisure time, occupational, and commuting physical activity and the risk of stroke. Stroke, 36(9), 1994-1999. doi: https://doi.org/10.1161/01.STR.0000177868.89946.0c

Hu, G., Tuomilehto, J., Silventoinen, K., Barengo, N., y Jousilahti, P. (2004). Joint effects of physical activity, body mass index, waist circumference and waist-to-hip ratio with the risk of cardiovascular disease among middle-aged Finnish men and women . European Heart Journal, 25, 2212-2219

Janssen, I., Katzmarzyk, P. T., y Ross, R. (2005). Body mass index is inversely related to mortality in older people after adjustment for waist circumference. J Am Geriatr Soc, 53(12), 2112-2118.

Johnson, N. P., Wu, E., Bonow, R. O., Holly, T. A. (2008). Relation of exercise capacity and body mass index to mortality in patients with intermediate to high risk of coronary artery disease. Am J Cardiol, 102(8), 1028-1033.

Kline, G. M., Porcari, J. P., y Hintermeister, R. (1987). Estimation of $\mathrm{VO}_{2}$ max from a one-mile track walk, gender, age, and body weight. Med Sci Sports Exerc, 19(3), 253-259. 
Kwon, I., Choi, S., Mittman, B., Bharmal, N., Liu, H., Vickrey, B.,...Sarkisian, C. (2015). Study protocol of "Worth the Walk": a randomized controlled trial of a stroke risk reduction walking intervention among racial/ethnic minority older adults with hypertension in community senior centers. BMC Neurol, 15, 91. doi: https://doi.org/10.1186/s12883-015-0346-9

Lee, I. M., Hsieh, C. C., y Paffenbarger, R. S., Jr. (1995). Exercise intensity and longevity in men. The Harvard Alumni Health Study. JAMA, 273(15), 1179-1184.

Leyk, D., Rohde, U., Gorges, W., Ridder, D., Wunderlich, M., Dinklage, C.,...Essfeld, D. (2006). Physical performance, body weight and BMI of young adults in Germany 2000 - 2004: results of the physical-fitness-test study. Int J Sports Med, 27(8), 642-647. doi: https://doi.org/10.1055/s-2005-872907

Malmberg, J., Miilunpalo, S., Pasanen, M., Vuori, I., y Oja, P. (2005). Characteristics of leisure time physical activity associated with risk of decline in perceived health--a 10-year follow-up of middle-aged and elderly men and women. Prev Med, 41(1), 141-150. doi: https://doi.org/10.1016/j.ypmed.2004.09.036

Manson, J. E., Greenland, P., LaCroix, A. Z., Stefanick, M. L., Mouton, C. P., Oberman, A.,...Siscovick, D. S. (2002). Walking compared with vigorous exercise for the prevention of cardiovascular events in women. N Engl J Med, 347(10), 716-725. doi: https://doi.org/10.1056/NEJMoa021067

Marfell-Jones, M., Olds, T., Stewart, A., y Carter, L. (2006). International standards for anthropometric assessment (ISAK Ed.). Potchefstroom, South Africa.

MinSalud. (1993). Resolución No. 008430 de 1993 - Ministerio de Salud de la República de Colombia. Recuperado de https://www.unisabana.edu.co/fileadmin/Documentos/Investigacion/comite de etica/Res 84301993 - Salud.pdf.

MinSalud. (2014). Análisis de situación de Salud. Colombia, 2014 - Ministerio de Salud y Protección Social. Recuperado de https://www.minsalud.gov.co/sites/rid/Lists/BibliotecaDigital/RIDE/VS/ED/PSP/ASIS 2014 v11.pdf

Morikawa, M., Okazaki, K., Masuki, S., Kamijo, Y., Yamazaki, T., Gen-no, H.,...Nose, H. (2011). Physical fitness and indices of lifestyle-related diseases before and after interval walking training in middle-aged and older males and females. Br J Sports Med, 45(3), 216-224. doi: https://doi.org/10.1136/bjsm.2009.064816

Mott, J. W., Wang, J., Thornton, J. C., Allison, D. B., Heymsfield, S. B., y Pierson, R. N., (1999). Relation between body fat and age in 4 ethnic groups. Am J Clin Nutr, 69(5), 1007-1013.

Ordaz Romay, E. (2006). Transformation of equations in analysis of proportionality through referent models (U. C. d. Madrid Ed.). Madrid.

Osei-Tutu, K. B., y Campagna, P. D. (2005). The effects of short- vs. long-bout exercise on mood, VO2max, and percent body fat. Prev Med, 40(1), 92-98. doi: https://doi.org/10.1016/j.ypmed.2004.05.005

Pahor, M., Blair, S. N., Espeland, M., Fielding, R., y Gill, T. M. (2006). Effects of a physical activity intervention on measures of physical performance: Results of the lifestyle interventions and independence for Elders Pilot (LIFE-P) study. J Gerontol A Biol Sci Med Sci, 61(11), 1157-1165

Paniagua, L., Lohsoonthorn, V., Lertmaharit, S., Jiamjarasrangsi, W., y Williams, M. A. (2008). Comparison of waist circumference, body mass index, percent body fat and other measure of adiposity in identifying cardiovascular disease risks among Thai adults. Obes Res Clin Pract, 2(3), 215-223.

Pate, R. R., Pratt, M., Blair, S. N., Haskell, W. L., Macera, C. A., y Bouchard, C. (1995). Physical activity and public health. A recommendation from the Centers for Disease Control and Prevention and the American College of Sports Medicine. JAMA, 273(5), 402-407.

Powell, K. E., y Paffenbarger, R. S., Jr. (1985). Workshop on Epidemiologic and Public Health Aspects of 
Physical Activity and Exercise: a summary. Public Health Rep, 100(2), 118-126.

Stewart, A. D. (2010). Kinanthropometry and body composition: a natural home for three-dimensional photonic scanning. J Sports Sci, 28(5), 455-457. doi: https://doi.org/10.1080/02640411003661304

Sui, X., LaMonte, M. J., Laditka, J. N., Hardin, J. W., Chase, N., y Hooker, S. P. (2007). Cardiorespiratory fitness and adiposity as mortality predictors in older adults. JAMA, 298(21), 2507-2516.

Thompson, P. D., Buchner, D., Pina, I. L., Balady, G. J., Williams, M. A., Marcus, B. H.,...Metabolism Subcommittee on Physical, A. (2003). Exercise and physical activity in the prevention and treatment of atherosclerotic cardiovascular disease: a statement from the Council on Clinical Cardiology (Subcommittee on Exercise, Rehabilitation, and Prevention) and the Council on Nutrition, Physical Activity, and Metabolism (Subcommittee on Physical Activity). Circulation, 107(24), 3109-3116. doi: https://doi.org/10.1161/01.CIR.0000075572.40158.77

Trivedi, T., Liu, J., Probst, J., Merchant, A., Jhones, S., y Martin, A. B. (2015). Obesity and obesity-related behaviors among rural and urban adults in the USA. Rural Remote Health, 15(4), 3267.

Vuillemin, A., Boini, S., Bertrais, S., Tessier, S., Oppert, J. M., Hercberg, S.,...Briancon, S. (2005). Leisure time physical activity and health-related quality of life. Prev Med, 41(2), 562-569. doi: https://doi.org/10.1016/j.ypmed.2005.01.006

WHO. (2006). World Health Organization. Body Mass Index Classification. Recuperado de http://apps.who.int/bmi/index.jsp?introPage=intro 3.html

WHO. (2004). World Health Organization. Appropriate body-mass index for Asian populations and its implications for policy and intervention strategies. Lancet, 363(9403), 157-163.

Wing, R. R. (1999). Physical activity in the treatment of the adulthood overweight and obesity: current evidence and research issues. Med Sci Sports Exerc, 31(11 Suppl), S547-552.

WMA. (2014). The Helsinki Declaration of the World Medical Association (WMA). Ethical principles of medical research involving human subjects. Pol Merkur Lekarski, 36(215), 298-301.

Wu, C. H, Heshka, S., Wang, J., Pierson, R. N., Jr., Heymsfield, S. B., y Laferrere, B. (2007). Truncal fat in relation to total body fat: influences of age, sex, ethnicity and fatness. Int J Obes, 31(9), 1384-1391.

Yuhasz, M. S. (1977). Physical fitness manual (U. o. W. Ontario Ed.). Canadá. 